\title{
Metformin and its anti-inflammatory and anti-oxidative effects; new concepts
}

\author{
Ali Hasanpour Dehkordi ${ }^{\circledR}$, Abolfazl Abbaszadeh $^{2}$, Samareh Mir $^{3}$, Amin Hasanvand ${ }^{4 *}$ \\ ${ }^{1}$ Department of Medical-Surgical, Faculty of Nursing and Midwifery, Shahrekord University of Medical Sciences, Shahrekord, Iran \\ ${ }^{2}$ Department of Surgery, Lorestan University of Medical Sciences, Khorramabad, Iran \\ ${ }^{3}$ Department of Pharmacotherapy, Faculty of pharmacy, Lorestan University of Medical Sciences, Khorramabad, Iran. \\ ${ }^{4}$ Nutritional Health Research Center, Department of Pharmacology and Toxicology, Faculty of Pharmacy, Lorestan University of Medical \\ Sciences, Khorramabad, Iran
}

\section{A R T I C L E I N F O}

Article Type:

Review

\section{Article History:}

Received: 9 May 2017

Accepted: 1 September 2018

Published online: 6 September 2018

Keywords:

Metformin

Oxidative stress

Inflammation

AMPK

\begin{abstract}
A B S T R A C T
Metformin is one of the oldest and commonly used blood sugar lowering drugs, having limited side effects and used as the first line treatment in patients suffering from diabetes mellitus. Moreover, various studies have emphasized on the anti-inflammatory and antioxidant role of metformin, with multiple mechanisms, which activation of AMPK by metformin has had a key role in many of them. During the searches on the internet websites of PubMed, Elsevier, Google Scholar, and Science Direct, 76 papers related to the anti-inflammatory and antioxidant role of metformin were selected and reviewed since 2003 to 2017. At the cellular level, metformin suppresses the inflammation in many cases and reduces or eliminates inflammatory factors mainly through dependent mechanisms and sometimes independent of AMPK at the cellular level and through other ways at the systematic levels. It is also effective in reducing the level of oxidative stress factors by regulating the antioxidant system of the cell. All evidence suggests the antioxidant and anti-inflammatory role of metformin in various conditions. Metformin can be an appropriate treatment option for many diseases, which inflammatory processes and oxidative stress play a role in their pathogenesis.
\end{abstract}

Implication for health policy/practice/research/medical education:

Metformin, with the chemical name of 1,1-dimethylbiguanide hydrochloride, is in the first line of treatment of patients with type 2 diabetes. Metformin treatment reduced inflammatory markers such as IL-1 $\beta$, iNOS, and TNF- $\alpha$, and reduced the number of microglia cells, and accordingly, it inhibited the inflammatory response. It was indicated that metformin reduced levels of ROS and $\mathrm{NO}$ and increased the antioxidant system, such as SOD.

Please cite this paper as: Hasanpour Dehkordi A, Abbaszadeh A, Mir S, Hasanvand A. Metformin and its anti-inflammatory and anti-oxidative effects; new concepts. J Renal Inj Prev. 2019;8(1):54-61. DOI: 10.15171/jrip.2019.11.

\section{Introduction}

Metformin, with chemical name of 1,1-dimethylbiguanide hydrochloride, is in the first line of treatment of patients with type 2 diabetes. Side effects of this drug are limited. The most important of them is increasing the blood lactate levels, its use has been limited in people with chronic kidney disease $(\mathrm{CKH})$, liver and heart failure (HF) (1). It seems that metformin to be effective in reducing the production of glucose from liver cells (gluconeogenesis), reducing insulin resistance, reducing fasting plasma insulin level, and activating the absorption of peripheral blood glucose by adenosine monophosphate-activated protein kinase (AMPK) (2-5). AMPK is an important factor in regulating the energy balance and its activation is associated with increasing the ratio of AMP to ATP. This enzyme plays various roles in different diseases $(6,7)$. Clinical studies have proven the protective effect of this drug in various tissues, including reduced stroke and thrombotic risk factors, death caused by cardiovascular diseases, endothelial cell inflammation, the proliferation of aorta smooth muscle cells, and anti-teratogenic property (2-4). Additionally, various studies have revealed the role of metformin in anti-inflammatory, anti-apoptotic, and antioxidant processes (8-10). Various studies have proposed different mechanisms for anti-inflammatory and antioxidant role of metformin, such as increasing the insulin level, followed by suppressing the inflammatory processes through decreasing blood glucose and regulating 
the molecules involved in inflammation $(11,12)$.

Materials and Methods

For this review, we used a variety of sources including PubMed, Embase, Scopus and directory of open access journals (DOAJ). The search was conducted by using combinations of the following key words and or their equivalents; metformin, oxidative stress, inflammation, AMPK.

\section{Anti-inflammatory role of metformin}

Metformin and pre-inflammatory transcription factors

The nuclear factor kappa B (NF-kB) is a transcription regulator involved in inflammation. By regulating the signaling, this factor can disrupt the process of several inflammatory pathways, cell death, and tissue destruction (13). In an examination conducted on mice with traumatic spinal cord injury, it was revealed that complex reactions of local inflammation along with microglia proliferation and activation, infiltration of phagocyte and increased production of pre-inflammatory cytokines occur in such conditions (14). Metformin can restrict the neuronal damage and disabilities caused by it during the early days through applying its anti-inflammatory effect by reducing the expression of NF-kB $(2,7)$. By reducing the phosphorylation of signal transducer and activator of transcription (STAT) through increasing the activation of AMPK, metformin inhibits the differentiation of monocyte into macrophages (15). In a research conducted in 2006, it was shown that metformin inhibited the expression of NF-kB-dependent genes, such as preinflammatory molecules and adhesion molecules such as intercellular adhesion molecule-1 (ICAM-1), vascular cell adhesion molecule-1(VCAM-1), selectin E, and monocyte chemoattractant protein-1 (MCP-1) (7). It has been shown that metformin inhibits the production of IL- 8 and IL-1 $\alpha$ inflammatory cytokines significantly by inhibiting the activation of NF-kB and phosphorylation of inhibitor of kappa B (IkB) in the intestinal epithelial cells of mice with acute colitis (16). Metformin also suppresses the expression of CXCL8 induced by lipopolysaccharide (LPS) by inhibiting NF-kB. This cytokine contributes in changing the microenvironment around the tumor by calling leukocytes and endothelial progenitors involved in angiogenesis (17).

In a research conducted to evaluate the anti-fibrotic effects of metformin in heart muscle cells, it was revealed that aldosterone can increase the expression of proinflammatory mediators of the TRAF3 interacting protein (TRAF3IP2) signaling pathway and the production of inflammatory cytokines such as IL-6, IL-17 and IL-18 through inducing oxidative stress $(5,18,19)$. TRAF3IP2 is a cytoplasmic adapter molecule responding to oxidative stress, which induces production of several transcription factors such as NF-kB, AP-1, and $\mathrm{C} / \mathrm{EBP} \beta$, and it is involved in the expression of inflammatory mediators. Investigations have indicated that metformin can inhibit the TRAF3IP2 molecule activated by aldosterone through activating the AMPK. It is also able to target the NF-kB or $\mathrm{C} / \mathrm{EBP} \beta$ transcription factors activation pathway, leading to reduced or stopped production of the inflammatory cytokines. Finally, proliferation and migration of cardiac fibroblasts are reduced and the risk of cardiac fibrosis is moderated (5,20-22). Metformin applies its antiinflammatory effect in atherosclerosis by inhibiting the phosphorylation of transcription factors P38, JNK and AKT induced by IL- 1 in smooth muscle cells. It also reduces the production of IL- 6 and IL-8 (cytokines responsible for calling monocytes and adhesion of epithelial cells) from epithelial cells and macrophages (23).

\section{Metformin and inflammatory markers}

Various studies have indicated different impacts of metformin on C-reactive protein (CRP) (24-26). CRP is one of the most important inflammatory markers and plays an important role in the pathogenesis of cardiovascular diseases, obesity, insulin resistance syndrome and polycystic ovary. In a study conducted with large sample size, metformin reduced CRP level after 24 weeks, but the mechanism of its action was not accurately determined $(4,27)$. In a meta-analysis carried out on 20 studies involving 433 women with polycystic ovary syndrome, the decreasing effect of metformin on CRP plasma levels, especially in obese women, was confirmed (28). In another meta-analysis conducted on 216 studies, it was found that metformin significantly reduced CRP in women with polycystic ovary syndrome, but it did not significantly affect the IL-6 (29).

Tumor necrosis factor-alpha (TNF- $\alpha$ ) is an important inflammatory cytokine, produced as a result of bacterial and viral infections in the body and can cause tissue damage and fibrosis (30). Cyclooxygenase-2 (COX-2) is also an essential protein involved in the production of prostaglandin during acute and chronic inflammation (31). In an investigation on the anti-inflammatory effects of metformin on inflammation caused by LPS in human middle ear epithelial cell lines (HMEECs), it was shown that the effect of LPS increases the levels of TNF- $\alpha$ and COX-2. By assessing the values of these factors with realtime polymerase chain reaction (PCR) and western blot method, they realized that pre-treatment with metformin could suppress the production of these inflammatory factors (30). In experimental arthritis, dose-dependent metformin could reduce the production and secretion of TNF- $\alpha$ and IL- 6 and moderate the inflammation through AMPK activation (32). Likewise, in another research, it was revealed that metformin could reduce the concentration of inflammatory marker YKL-40 (33). Metformin also reduces the production of sepsis inflammatory cytokines, produced by neutrophils and monocytes, including IL6 , IL-1 $\beta$, and TNF- $\alpha$ (34). In an investigation on obese children aged 6 to 12 years, it was found that metformin reduced the level of inflammatory factors such as TNF- $\alpha$, IL-8 and insulin resistance (35). In the ischemicreperfusion model, it was shown that metformin reduced the expression of induced pre-inflammatory factors such 
as TNF- $\alpha$, IL-1 $\beta$, TLR4 and Ccr2, and the penetration of monocytes and macrophages (36).

The process of obesity is associated with increased oxidative and pro-inflammatory factors. Migratory inhibitor factor (MIF) is one of the most important inflammatory factors, secreted from adipose tissue and cells such as macrophage and monocyte. It can be involved in forming the sclerotic plaques. Its value is also increased in people with type 2 diabetes and it is an essential mediator for initiating the acquired immune responses. An investigation conducted in 2004 revealed that the effect of metformin on obese people significantly reduced the expression of mRNA and secretion of MIF, and the anti-inflammatory role of metformin and its effect on reducing the atherosclerotic processes were observed (37).

Advanced glycation end products (AGEs) are one of the most important inflammatory factors in diabetes, affecting the atherosclerosis development. With an effect on macrophages, these factors exacerbate the expression of pro-inflammatory cytokines (IL-1, IL-6 and TNF- $\alpha$ ), increase the RAGE expression, and activate NF- $\beta B$ pathway. In fact, RAGE/NF-kB signaling plays role in the inflammatory activity of AGE-stimulated macrophages (38). Activating the AMPK and inhibiting the NF-kB, metformin suppresses the pathway of RAGE/ NF-kB, leading to inhibited effects of AGE and change of phenotype of macrophages from M1 (classical or inflammatory) to M2 (alternatives) by changing the expression of their surface markers (CD86 and CD206, respectively). Finally, the production of inflammatory cytokines and inhibitory cytokines (IL-10) would increase (39). By producing the high mobility group box 1 (HMGB1), which has pseudo-cytokine activity, necrotic cells are able to stimulate many receptors, including TLR4 and RAGE and induce inflammatory responses. Metformin can be attached directly to the extracellular HMGB1 on C-terminal sequence and inhibit it (40). Inflammation plays a crucial role in the death of dopaminergic neurons and development of Parkinson disease as a result (3). The change in ATP mitochondrial production in this disease can damage the neurons and production of danger-associated molecular patterns (DAMP). Identifying the DAMPs by microglia would result in initiating the inflammatory and neurotoxic activities (41). Metformin can pass through the bloodbrain barrier and affect the central nervous system. Recent studies confirmed the reducing role of metformin on the activity of microglia cells $(42,43)$. Metformin injection in the Parkinson's animal model reduced inflammatory markers such as IL-1 $\beta$, iNOS, and TNF- $\alpha$, and reduced the number of microglia cells, and accordingly, it inhibited the inflammatory response (44). However, metformin is not able to reduce the death of neuronal cells (3).

\section{Metformin and regulation of cell differentiation}

Multiple sclerosis (MS) is one of the autoimmune inflammatory diseases, which its accurate mechanism of action has not been fully understood. Investigations suggest that TH17 cells response against myelin nerves and dysfunction of Treg cells are very effective in its pathogenesis. In the EAE mouse model of this disease, the accumulation of TH17 cells was seen in the central nervous system. They are involved in pathogenesis of this disease by the production of IL-17 followed by inducing the production of other pre-inflammatory cytokines such as IL-1 and IL-6 (45). In a research conducted in 2016 to investigate the role of metformin in regulating the TH17/Treg balance in the EAE mouse model and several other studies, it was shown that injecting the metformin can improve the clinical signs of EAE. These studies revealed that metformin suppressed the expression of mammalian target of rapamycin (mTOR) and STAT3 transcription factors and the factors regulating differentiation and activation of TH17 cells by activating AMPK, and thereby, it prevented the proliferation and penetration of these cells into central nervous system (CNS). In addition, by activating AMPK, metformin can increase the proliferation of Treg cells and increase their infiltration to the central nervous tissue, and induce the production of anti-inflammatory cytokines such as IL10 and TGF- $\beta$ from these cells, and thereby, it changes the balance of TH17/Treg by more production of Treg and reduction of TH17 $(45,46)$. Another mechanism involved in regulating this cellular balance by metformin is its effect on metabolic pathways. Th17 cells, compared to Treg, require more energy to apply its effect and they obtain their energy through pathways such as aerobic glycolysis and glutamine catabolism. Signaling mTOR and its downstream genes are involved in inducing these metabolic pathways, while Treg cells obtain their energy through lipid oxidation $(47,48)$. By increasing the activation of AMPK in the liver cells, followed by MTOR inhibition, metformin causes induction of the genes involved in lipid metabolism, reduced glycolysis and glutamine catabolism, and thereby, it reduces the TH17 cells and reduces the inflammation (49). In an investigation conducted in 2016 on intraperitoneal injection of metformin in patients with acute graft-versus-host disease ( $a$ GVHD), it was shown that the activation of AMPK signaling reduces the severity of this reaction. Due to the effect of metformin, STAT3 activity is reduced, mTOR/STAT3 signaling is inhibited, leading to induction of autonomy, and it changes the TH17/Treg cellular balance toward more production of Treg cells (50). In addition, in the SLE mouse model, oral injection of metformin inhibited the differentiation of B cells from plasmas cells and changed the process of production of autoantibody, inhibited the formation of germ cells, and penetration of inflammatory cells into tissues such as the liver and kidney, by regulating the AMPK-mTOR-STAT3 signaling pathway. These changes were associated with inhibiting the differentiation of $\mathrm{T}$ cells into inflammatory subgroups such as TH17 and TFH and increasing the differentiation to Treg (51).

The antioxidant role of metformin

Hyperglycemia induces the oxidative stress. An 
investigation conducted on 208 patients with diabetes mellitus suggests increased oxidative stress markers such as advanced oxidation protein product (AOPP), pentosidine, and reactive oxygen species (ROS) in these people. In addition, the level of nitric oxide, as an important mediator in nerve, immune and cardiovascular systems, decreased. After injecting the metformin in these people, level of oxidative stress indices decreased and the nitric oxide levels significantly increased (52).

ROS are produced in the mitochondrial electron transfer chain and cause production of ATP from ATP synthase. During these inflammatory processes, such as bacterial infection and stimulation with LPS, the production of these oxidants increases. Studies have revealed that administration of metformin stops the production of free oxygen radicals by direct inhibiting of the complex I electron transfer complex chain (NADH ubiquitin oxidoreductase (NADH) (30,53).

Inhibition of complex I chain reduces the production of ATP and increases the ratio of ADP/ATP and AMP/ATP, which is the main stimulant for activation of the AMPK. This complex is involved in inducing the production of IL- $1 \beta$ through ROS. By blocking the complex I, metformin inhibited the production of IL-1 $\beta$ induced by LPS and increased the production of IL-10 (54). It was also revealed that metformin can apply its antioxidant effects by inhibiting NAD (P)H/PKC oxidase pathways (55). In an investigation conducted to evaluate the antioxidant role of metformin in the oxidative damage induced mouse model in the liver by carbon tetrachloride, it was found that the level of liver aminotransferases was reduced and its histopathological characteristics were improved following treatment with metformin. The dose-dependent metformin also reduced the level of liver peroxide hydrogen (56).

Stimulating the mineralocorticoid receptors and NOX4, aldosterone increases the production of $\mathrm{H} 2 \mathrm{O} 2$ in cardiac fibroblasts, followed by inducing the expression of the cytoplasmic adapter TRAF3IP2. In these conditions, metformin inhibits oxidative reactions by applying its antioxidant effects. Metformin can play its role through several different mechanisms, including 1) direct trapping of hydroxyl radicals; 2) activating antioxidant enzymes such as catalase, which is the main decomposer of $\mathrm{H} 2 \mathrm{O} 2$; and 3) reducing the transcription from NOX4 in longterm injection of metformin $(5,57,58)$.

TGF- $\beta$ is one of the most important inflammatory factors, stimulating the production of ROS, accumulation of inflammatory cells in the lung, pulmonary fibrosis in mice with asthma by activating the Sma mothers against decapentaplegic (SMAD2/3) and mitogen-activated protein kinase (MAPK) signaling pathways $(59,60)$. Activating the AMPK and inhibiting the TGF- $\beta$ signaling and inducing endogen antioxidant system including glutathione reductase (GSH), superoxide dismutase (SOD) and catalase (CAT), metformin reduces the ROS and malondialdehyde (61-63). In a research conducted by Hyun et al on an animal model of peritoneal dialysis, it was shown that by independent and dependent AMPK mechanisms, metformin can reduce ROS production, inhibit NADPH oxidase (NOX) activation, and exacerbate antioxidant activity and SOD expression in mesothelial cells (64). In addition, metformin activated the transcription factor of SKN-1/Nrf2 and increased the expression of antioxidant genes in animal models (65). In the process of sepsis pathogenesis, the abundant production of inflammatory cytokines leads to increased production of cytokines and ROS by leaving an effect on microglia and endothelial cells. This oxidative stress can be involved in the onset of brain damages. Investigations have indicated that metformin leads to AKT phosphorylation by phosphatidylinositol-3-kinase (PI3K) activation, which this process is involved in maintaining mitochondrial integrity and increasing the ability to cope with damage induced by inflammation and oxidative stress. By inducing the production and increasing the activity of the antioxidant system, such as SOD, metformin cleans the ROS from brain tissue $(34,66,67)$.

During the brain ischemia, ROS production increases in tissue. In these conditions, the value of antioxidant nuclear factor erythroid 2-related factor (Nrf2), which is an oxidative stress sensor, and hexokinase increase in the hippocampus, and then, decrease in time-dependent form. In addition, AMP and ATP levels increase and decrease, respectively, under ischemia and it acts as a stimulant for AMPK activity. AMPK is able to stabilize Nrf2 and induce its gene expression. The induction of the Nrf2 pathway is associated with increasing level of antioxidant system enzymes of body such as CAT, GSH and SOD. In this regard, by induction of AMPK activation, metformin stimulates the onset of this pathway and applies its antioxidant role (44).

Various studies have emphasized the major role of oxidative stress and consequently increase in the indicators resulting from the oxidation of lipids and proteins in the early phase of Alzheimer's disease. In an investigation conducted by Chen and Zhong on Alzheimer disease, it was shown that the oxidation of proteins was associated with reduced glucose metabolism in brain cells, followed by disorder in glucose humoral and neuronal dysfunction $(68,69)$. Moreover, increasing the reactive nitrogen species (RNS) in animal models might cause beta-amyloid deposition in the brain tissue, which might lead into electron transfer chain disruption, mitochondrial dysfunction, and increased ROS production (70). The AMPK activation by metformin induces autophagy and decomposes the betaamyloid and improves the neurological changes related to Alzheimer's disease (71).

In an investigation conducted in 2016, it was indicated that metformin reduced levels of ROS and NO in mouse adipose-derived stem cells (MuASCs) and increased the antioxidant system, such as SOD (72). By reducing the level of ROS and MDA and increasing the production and activity of GSH, metformin also can inhibit the oxidative stress associated with arsenic and butyric acid (73). In another study, it was revealed that oxidative stress 
markers were reduced in the culture of mouse olfactory ensheathing cells (mOECs), which received metformin (74). Algire et al indicated that metformin, independent of AMPK, reduced the production of ROS resulting from the effect of paraquat and prevented DNA damage and mutation (75). In another investigation on the stroke experimental model, metformin injection reduced the level of ROS by regulating antioxidant activity (76).

\section{Conclusion}

Numerous experimental studies have been conducted to evaluate the role of metformin in inhibiting inflammatory processes and oxidative stress, but limited clinical studies have been conducted in this regard. It has shown different effects in vitro and in vivo conditions. Moreover, the mechanism of action of this drug has been different under various conditions. By examining the process of inflammatory and oxidative diseases and metformin role in controlling or changing these pathways in clinical and laboratory conditions, we hope that this drug with fewer side effects to be used in the treatment of many diseases.

\section{Authors' contribution}

AHD, AA and SM searched the data and prepared the draft of the manuscript. AH edited and finalized the paper. All authors read and signed the final manuscript.

\section{Conflicts of interest}

The authors declared no competing interests.

\section{Ethical considerations}

Ethical issues (including plagiarism, data fabrication, double publication) have been completely observed by the authors.

\section{Funding/Support}

None.

\section{References}

1. Nesti L, Natali A. Metformin effects on the heart and the cardiovascular system: A review of experimental and clinical data. Nutr Metab Cardiovasc Dis. 2017;27:657-669. doi: 10.1016/j.numecd.2017.04.009.

2. Wang C, Liu C, Gao K, Zhao H, Zhou Z, Shen Z, et al. Metformin preconditioning provide neuroprotection through enhancement of autophagy and suppression of inflammation and apoptosis after spinal cord injury. Biochem Biophys Res Commun. 2016 2;477:534-540. doi: 10.1016/j.bbrc.2016.05.148.

3. Ismaiel AA, Espinosa-Oliva AM, Santiago M, GarcíaQuintanilla A, Oliva-Martín MJ, Herrera AJ, et al. Metformin, besides exhibiting strong in vivo antiinflammatory properties, increases mptp-induced damage to the nigrostriatal dopaminergic system. Toxicol Appl Pharmacol. 2016;298:19-30. doi: 10.1016/j. taap.2016.03.004.

4. Carter A, Bennett C, Bostock J, Grant P. Metformin reduces C-reactive protein but not complement factor C3 in overweight patients with type 2 diabetes mellitus. Diabetic medicine. 2005;22:1282-4.

5. Mummidi S, Das NA, Carpenter AJ, Kandikattu H, Krenz M, Siebenlist U, et al. Metformin inhibits aldosterone-induced cardiac fibroblast activation, migration and proliferation in vitro, and reverses aldosterone+ salt-induced cardiac fibrosis in vivo. J Mol Cell Cardiol. 2016;98:95-102. doi: 10.1016/j.yjmcc.2016.07.006.

6. Grahame Hardie D. AMP-activated protein kinase: a key regulator of energy balance with many roles in human disease. J Intern Med. 2014;276:543-59. doi: 10.1111/ joim. 12268 .

7. Hattori Y, Suzuki K, Hattori S, Kasai K. Metformin inhibits cytokine-induced nuclear factor $\mathrm{\kappa B}$ activation via AMPactivated protein kinase activation in vascular endothelial cells. Hypertension. 2006;47:1183-8.

8. Nath N, Khan M, Paintlia MK, Hoda MN, Giri S. Metformin attenuated the autoimmune disease of the central nervous system in animal models of multiple sclerosis. J Immunol. 2009;15;182:8005-14. doi: 10.4049/jimmunol.0803563.

9. Ullah I, Ullah N, Naseer MI, Lee HY, Kim MO, Neuroprotection with metformin and thymoquinone against ethanol-induced apoptotic neurodegeneration in prenatal rat cortical neurons. BMC Neurosci. 2012;19;13:11. doi: $\quad 10.1186 / 1471-2202-13-11$.

10. Alzoubi H, Khabour F, I Al-azzam S, H Tashtoush M, M Mhaidat N. Metformin eased cognitive impairment induced by chronic L-methionine administration: potential role of oxidative stress. Curr Neuropharmacol. 2014;12:186-92. doi: 10.2174/1570159X11666131120223201.

11. Hyun E, Ramachandran R, Hollenberg MD, Vergnolle N. Mechanisms behind the anti-inflammatory actions of insulin. Crit Rev Immunol. 2011;31:307-40.

12. Monnier L, Hanefeld M, Schnell O, Colette C, Owens D. Insulin and atherosclerosis: how are they related? Diabetes Metab. 2013;39:111-7. doi: 10.1016/j.diabet.2013.02.001.

13. Brambilla R, Hurtado A, Persaud T, Esham K, Pearse DD, Oudega $\mathrm{M}$, et al. Transgenic inhibition of astroglial NF- $\mathrm{KB}$ leads to increased axonal sparing and sprouting following spinal cord injury. J Neurochem. 2009;110:765-78. doi: 10.1111/j.1471-4159.2009.06190.x.

14. Alexander JK, Popovich PG. Neuroinflammation in spinal cord injury: therapeutic targets for neuroprotection and regeneration. Prog Brain Res. 2009;175:125-37. doi: 10.1016/S0079-6123(09)17508-8.

15. Vasamsetti SB, Karnewar S, Kanugula AK, Thatipalli AR, Kumar JM, Kotamraju S. Metformin inhibits monocyte-tomacrophage differentiation via AMPK-mediated inhibition of STAT3 activation: potential role in atherosclerosis. Diabetes. 2015;64:2028-41. doi: 10.2337/db14-1225.

16. Koh SJ, Kim JM, Kim IK, Ko SH, Kim JS. Antiinflammatory mechanism of metformin and its effects in intestinal inflammation and colitis-associated colon cancer. J Gastroenterol Hepatol. 2014;29:502-10.

17. Xiao Z, Wu W, Poltoratsky V. Metformin Suppressed CXCL8 Expression and Cell Migration in HEK293/TLR4 Cell Line. Mediators Inflamm. 2017;2017:6589423. doi: $10.1155 / 2017 / 6589423$.

18. Sakamuri SS, Valente AJ, Siddesha JM, Delafontaine P, Siebenlist U, Gardner JD, et al. TRAF3IP2 mediates aldosterone/salt-induced cardiac hypertrophy and fibrosis. Mol Cell Endocrinol. 2016;5;429:84-92. doi: 10.1016/j. mce.2016.03.038.

19. Baldeviano GC, Barin JG, Talor MV, Srinivasan S, Bedja 
$\mathrm{D}$, Zheng $\mathrm{D}$, et al. Interleukin-17A is dispensable for myocarditis but essential for the progression to dilated cardiomyopathy. Circ Res. 2010;28;106:1646-55. doi: 10.1161/CIRCRESAHA.109.213157.

20. Liu X-m, Peyton KJ, Shebib AR, Wang H, Korthuis RJ, Durante W. Activation of AMPK stimulates heme oxygenase-1 gene expression and human endothelial cell survival. Am J Physiol Heart Circ Physiol. 2011;300:H8493. doi: 10.1152/ajpheart.00749.2010.

21. Somanna NK, Yariswamy M, Garagliano JM, Siebenlist U, Mummidi S, Valente AJ, et al. Aldosterone-induced cardiomyocyte growth, and fibroblast migration and proliferation are mediated by TRAF3IP2. Cell Signal. 2015;27:1928-38. doi: 10.1016/j.cellsig.2015.07.001.

22. Valente AJ, Sakamuri SS, Siddesha JM, Yoshida T, Gardner JD, Prabhu R, et al. TRAF3IP2 mediates interleukin-18-induced cardiac fibroblast migration and differentiation. Cell Signal. 2013;25:2176-84. doi: 10.1016/j. cellsig.2013.07.013.

23. Isoda K, Young JL, Zirlik A, MacFarlane LA, Tsuboi N, Gerdes N, et al. Metformin inhibits proinflammatory responses and nuclear factor- $\mathrm{\kappa B}$ in human vascular wall cells. Arterioscler Thromb Vasc Biol. 2006;26:611-617

24. Yatagai T, Nakamura T, Nagasaka S, Kusaka I, Ishikawa S-e, Yoshitaka A, et al. Decrease in serum C-reactive protein levels by troglitazone is associated with pretreatment insulin resistance, but independent of its effect on glycemia, in type 2 diabetic subjects. D Diabetes Res Clin Pract. 2004;63:1926.

25. Association $\mathrm{AD}$. Intensive lifestyle intervention or metformin on inflammation and coagulation in participants with impaired glucose tolerance. Diabetes. 2005;54:15661572.

26. Pradhan AD, Everett BM, Cook NR, Rifai N, Ridker PM. Effects of initiating insulin and metformin on glycemic control and inflammatory biomarkers among patients with type 2 diabetes: the LANCET randomized trial. JAMA. 2009;16;302:1186-94. doi: 10.1001/jama.2009.1347.

27. Morin-Papunen L, Rautio K, Ruokonen A, Hedberg P, Puukka M, Tapanainen JS. Metformin reduces serum C-reactive protein levels in women with polycystic ovary syndrome. J Clin Endocrinol Metab. 2003;88:4649-54.

28. Chen Y, Li M, Deng H, Wang S, Chen L, Li N, et al. Impact of metformin on C-reactive protein levels in women with polycystic ovary syndrome: a meta-analysis. Oncotarget. 2017;23;8:35425-35434. doi: 10.18632/oncotarget.16019.

29. Wang J, Zhu L, Hu K, Tang Y, Zeng X, Liu J, et al. Effects of metformin treatment on serum levels of C-reactive protein and interleukin-6 in women with polycystic ovary syndrome: a meta-analysis: A PRISMA-compliant article. Medicine. 2017;96:e8183. doi: 10.1097/MD.0000000000008183.

30. Cho JG, Song JJ, Choi J, Im GJ, Jung HH, Chae SW. The suppressive effects of metformin on inflammatory response of otitis media model in human middle ear epithelial cells. Int J Pediatr Otorhinolaryngol. 2016;89:28-32. doi: 10.1016/j.ijporl.2016.07.025.

31. Song J-J, Lim HW, Kim K, Kim K-M, Cho S, Chae S-W. Effect of caffeic acid phenethyl ester (CAPE) on $\mathrm{H} 2 \mathrm{O} 2$ induced oxidative and inflammatory responses in human middle ear epithelial cells. Int J Pediatr Otorhinolaryngol. 2012;76:675-9. doi: 10.1016/j.ijporl.2012.01.041.

32. Yan H, Zhou H-f, Hu Y, Pham CT. Suppression of experimental arthritis through AMP-activated protein kinase activation and autophagy modulation. J Rheum Dis Treat. 2015;28;1:5.

33. Esteghamati A, Rezvani S, Khajeh E, Ebadi M, Nakhjavani M, Noshad S. Comparative effects of metformin and pioglitazone on YKL-40 in type 2 diabetes: a randomized clinical trial. J Endocrinol Invest. 2014;37:1211-8. doi: 10.1007/s40618-014-0154-x.

34. Tang G, Yang H, Chen J, Shi M, Ge L, Ge X, et al. Metformin ameliorates sepsis-induced brain injury by inhibiting apoptosis, oxidative stress and neuroinflammation via the PI3K/Akt signaling pathway. Oncotarget. 2017;10;8:9797797989.

35. Dehkordi EH, Sattari F, Khoshdel A, Kasiri K. Effect of folic acid and metformin on insulin resistance and inflammatory factors of obese children and adolescents. J Res Med Sci. 2016;1;21:71.

36. Cahova M, Palenickova E, Dankova H, Sticova E, Burian M, Drahota Z, et al. Metformin prevents ischemia reperfusion-induced oxidative stress in the fatty liver by attenuation of reactive oxygen species formation. Am J Physiol Gastrointest Liver Physiol. 2015;15;309:G100-11. doi: 10.1152/ajpgi.00329.2014.

37. Dandona P, Aljada A, Ghanim H, Mohanty P, Tripathy C, Hofmeyer D, et al. Increased plasma concentration of macrophage migration inhibitory factor (MIF) and MIF mRNA in mononuclear cells in the obese and the suppressive action of metformin. J Clin Endocrinol Metab. 2004;89:5043-7.

38. Jin X, Yao T, Zhou Ze, Zhu J, Zhang S, Hu W, et al. Advanced glycation end products enhance macrophages polarization into M1 phenotype through activating RAGE/NF- $\kappa B$ pathway. Biomed Res Int. 2015;2015:732450.

39. Zhou Z, Tang Y, Jin X, Chen C, Lu Y, Liu L, et al. Metformin inhibits advanced glycation end products-induced inflammatory response in murine macrophages partly through AMPK activation and RAGE/NFkappaB Pathway suppression. J Diabetes Res. 2016;2016:4847812.

40. Horiuchi T, Sakata N, Narumi Y, Kimura T, Hayashi T, Nagano K, et al. Metformin directly binds the alarmin HMGB1 and inhibits its proinflammatory activity. J Biol Chem. 2017;292:8436-46.

41. Chen S-H, Oyarzabal EA, Hong J-S. Critical role of the Mac1/NOX2 pathway in mediating reactive microgliosisgenerated chronic neuroinflammation and progressive neurodegeneration. Curr Opin Pharmacol. 2016; 26: 54-60.

42. Towler MC, Hardie DG. AMP-activated protein kinase in metabolic control and insulin signaling. Circulation research. 2007;100(3):328-41.

43. Łabuzek K, Liber S, Gabryel B, Okopień B. Metformin has adenosine-monophosphate activated protein kinase (AMPK)-independent effects on LPS-stimulated rat primary microglial cultures. Pharmacol Rep. 2010;62:82748.

44. Ashabi G, Khalaj L, Khodagholi F, Goudarzvand M, Sarkaki A. Pre-treatment with metformin activates Nrf2 antioxidant pathways and inhibits inflammatory responses through induction of AMPK after transient global cerebral ischemia Metab Brain Dis. 2015;30:747-54. doi: 10.1007/ s11011-014-9632-2.

45. Sun Y, Tian T, Gao J, Liu X, Hou H, Cao R, et al. Metformin ameliorates the development of experimental autoimmune encephalomyelitis by regulating $\mathrm{T}$ helper 17 and regulatory T cells in mice. J Neuroimmunol. 2016;15;292:58-67. doi: 
10.1016/j.jneuroim.2016.01.014.

46. Nagai S, Kurebayashi Y, Koyasu S. Role of PI3K/Akt and mTOR complexes in Th17 cell differentiation. Ann N Y Acad Sci. 2013;1280:30-4. doi: 10.1111/nyas.12059.

47. Wang R, Green DR. Metabolic reprogramming and metabolic dependency in $\mathrm{T}$ cells. Immunol Rev. 2012;249:14-26. doi: 10.1111/j.1600-065X.2012.01155.x.

48. Michalek RD, Gerriets VA, Jacobs SR, Macintyre AN, MacIver NJ, Mason EF, et al. Cutting edge: distinct glycolytic and lipid oxidative metabolic programs are essential for effector and regulatory CD4+ T cell subsets. J Immunol. 2011;15;186:3299-303. doi: 10.4049/jimmunol.1003613.

49. Lee WH, Kim SG. AMPK-dependent metabolic regulation by PPAR agonists. PPAR Res. 2010;2010:549101. doi: $10.1155 / 2010 / 549101$.

50. Park M-J, Lee S-Y, Moon S-J, Son H-J, Lee S-H, Kim $\mathrm{E}-\mathrm{K}$, et al. Metformin attenuates graft-versus-host disease via restricting mammalian target of rapamycin/ signal transducer and activator of transcription 3 and promoting adenosine monophosphate-activated protein kinase-autophagy for the balance between $\mathrm{T}$ helper 17 and Tregs. Transl Res. 2016;173:115-130. doi: 10.1016/j. trsl.2016.03.006.

51. Lee SY, Moon SJ, Kim EK, Seo HB, Yang EJ, Son HJ, et al. Metformin Suppresses Systemic Autoimmunity in Roquin(san/san) Mice through Inhibiting B Cell Differentiation into Plasma Cells via Regulation of AMPK/ mTOR/STAT3. J Immunol. 2017;1;198:2661-2670. doi: 10.4049/jimmunol.1403088.

52. Chakraborty A, Chowdhury S, Bhattacharyya M. Effect of metformin on oxidative stress, nitrosative stress and inflammatory biomarkers in type 2 diabetes patients. Diabetes Res Clin Pract. 2011;93(1):56-62. doi: 10.1016/j. diabres.2010.11.030.

53. Diniz Vilela D, Gomes Peixoto L, Teixeira RR, Belele Baptista N, Carvalho Caixeta D, Vieira de Souza A, et al. The Role of Metformin in Controlling Oxidative Stress in Muscle of Diabetic Rats. Oxid Med Cell Longev. 2016;2016:6978625. doi: 10.1155/2016/6978625.

54. Kelly B, Tannahill GM, Murphy MP, O’Neill LA. Metformin inhibits the production of reactive oxygen species from NADH: ubiquinone oxidoreductase to limit induction of interleukin-1 $\beta$ (IL-1 $\beta$ ) and boosts interleukin-10 (IL10) in lipopolysaccharide (LPS)-activated macrophages. J Biol Chem. 2015;290(33):20348-59. doi: 10.1074/jbc. M115.662114.

55. Batchuluun B, Inoguchi $\mathrm{T}$, Sonoda $\mathrm{N}$, Sasaki $\mathrm{S}$, Inoue $\mathrm{T}$, Fujimura $Y$, et al. Metformin and liraglutide ameliorate high glucose-induced oxidative stress via inhibition of PKCNAD (P) H oxidase pathway in human aortic endothelial cells. Atherosclerosis. 2014;232:156-64. doi: 10.1016/j. atherosclerosis.2013.10.025.

56. Markowicz-Piasecka M, Sikora J, Szydłowska A, Skupień A, Mikiciuk-Olasik E, Huttunen KM. Metformin-a Future Therapy for Neurodegenerative Diseases. Pharm Res. 2017;34:2614-2627. doi: 10.1007/s11095-017-2199-y.

57. Bułdak Ł, Łabuzek K, Bułdak RJ, Kozłowski M, Machnik G, Liber S, et al. Metformin affects macrophages' phenotype and improves the activity of glutathione peroxidase, superoxide dismutase, catalase and decreases malondialdehyde concentration in a partially AMPKindependent manner in LPS-stimulated human monocytes/ macrophages. Pharmacol Rep. 2014;66:418-29. doi: 10.1016/j.pharep.2013.11.008.

58. Dai J, Liu M, Ai Q, Lin L, Wu K, Deng X, et al. Involvement of catalase in the protective benefits of metformin in mice with oxidative liver injury. Chem Biol Interact. 2014;5;216:34-42. doi: 10.1016/j.cbi.2014.03.013.

59. Ahn JY, Kim MH, Lim MJ, Park S, Yun YS, Song JY. The inhibitory effect of ginsan on TGF- $\beta$ mediated fibrotic process. J Cell Physiol. 2011;226(5):1241-7. doi: 10.1002/ jcp.22452.

60. Park CS, Bang B-R, Kwon H-S, Moon K-A, Kim T-B, Lee $\mathrm{K}-\mathrm{Y}$, et al. Metformin reduces airway inflammation and remodeling via activation of AMP-activated protein kinase. Biochem Pharmacol. 2012;15;84:1660-70. doi: 10.1016/j. bcp.2012.09.025.

61. Kandhare AD, Bodhankar SL, Mohan V, Thakurdesai PA. Effect of glycosides based standardized fenugreek seed extract in bleomycin-induced pulmonary fibrosis in rats: decisive role of Bax, Nrf2, NF- $\mathrm{kB}$, Muc5ac, TNF- $\alpha$ and IL1 $\beta$. Chem Biol Interact. 2015;25;237:151-65. doi: 10.1016/j. cbi.2015.06.019.

62. Ma J, Yu H, Liu J, Chen Y, Wang Q, Xiang L. Metformin attenuates hyperalgesia and allodynia in rats with painful diabetic neuropathy induced by streptozotocin. Eur J Pharmacol. 2015;5;764:599-606. doi: 10.1016/j. ejphar.2015.06.010.

63. Gamad N, Malik S, Suchal K, Vasisht S, Tomar A, Arava S, et al. Metformin alleviates bleomycin-induced pulmonary fibrosis in rats: Pharmacological effects and molecular mechanisms. Biomed Pharmacother. 2018;97:1544-53.

64. Shin HS, Ko J, Kim DA, Ryu ES, Ryu HM, Park SH, et al. Metformin ameliorates the Phenotype Transition of Peritoneal Mesothelial Cells and Peritoneal Fibrosis via a modulation of Oxidative Stress. Sci Rep. 2017;18;7:5690. doi: 10.1038/s41598-017-05836-6.

65. Onken B, Driscoll M. Metformin induces a dietary restriction-like state and the oxidative stress response to extend C. elegans healthspan via AMPK, LKB1, and SKN-1. PLoS One. 2010;18;5:e8758. doi: 10.1371/journal. pone. 0008758 .

66. Garabadu D, Krishnamurthy S. Metformin attenuates hepatic insulin resistance in type-2 diabetic rats through PI3K/Akt/GLUT-4 signalling independent to bicucullinesensitive GABAA receptor stimulation. Pharm Biol. 2017;55:722-728. doi: 10.1080/13880209.2016.1268635.

67. Xu H, Zhou Y, Liu Y, Ping J, Shou Q, Chen F, et al. Metformin improves hepatic IRS2/PI3K/Akt signaling in insulin-resistant rats of NASH and cirrhosis. J Endocrinol. 2016;229:133-44. doi: 10.1530/JOE-15-0409.

68. Butterfield DA, Poon HF, Clair DS, Keller JN, Pierce WM, Klein JB, et al. Redox proteomics identification of oxidatively modified hippocampal proteins in mild cognitive impairment: insights into the development of Alzheimer's disease. Neurobiol Dis. 2006;22:223-32.

69. Chen Z, Zhong C. Decoding Alzheimer's disease from perturbed cerebral glucose metabolism: implications for diagnostic and therapeutic strategies. Prog Neurobiol. 2013;108:21-43. doi: 10.1016/j.pneurobio.2013.06.004.

70. Reddy PH, Manczak M, Mao P, Calkins MJ, Reddy AP, Shirendeb U. Amyloid- $\beta$ and mitochondria in aging and Alzheimer's disease: implications for synaptic damage and cognitive decline. J Alzheimers Dis. 2010;20 Suppl 2:S499512. doi: 10.3233/JAD-2010-100504.

71. Zhang L, Zhang S, Maezawa I, Trushin S, Minhas P, Pinto 
M, et al. Modulation of mitochondrial complex I activity averts cognitive decline in multiple animal models of familial Alzheimer's disease. EBioMedicine. 2015;1;2:294305.

72. Marycz K, Tomaszewski KA, Kornicka K, Henry BM, Wroński S, Tarasiuk J, et al. Metformin decreases reactive oxygen species, enhances osteogenic properties of adiposederived multipotent mesenchymal stem cells in vitro, and increases bone density in vivo. Oxid Med Cell Longev. 2016;2016:9785890. doi: 10.1155/2016/9785890.

73. Ahangarpour A, Zeidooni L, Rezaei M, Alboghobeish S, Samimi A, Oroojan AA. Protective effect of metformin on toxicity of butyric acid and arsenic in isolated liver mitochondria and langerhans islets in male mice: an in vitro study. Iran J Basic Med Sci. 2017;20:1297-1305. doi: 10.22038/IJBMS.2017.9567.
74. Smieszek A, Strek Z, Kornicka K, Grzesiak J, Weiss C, Marycz K. Antioxidant and anti-senescence effect of metformin on mouse olfactory ensheathing cells (mOECs) may be associated with increased brain-derived neurotrophic factor levels-an ex vivo study. Int J Mol Sci. 2017;20;18. doi: 10.3390/ijms18040872.

75. Algire C, Moiseeva O, Deschênes-Simard X, Amrein L, Petruccelli L, Birman E, et al. Metformin reduces endogenous reactive oxygen species and associated DNA damage. Cancer Prev Res. 2012;5:536-43. doi: 10.1158/19406207.CAPR-11-0536.

76. Abd-Elsameea A, Moustaf A, Mohamed A. Modulation of the oxidative stress by metformin in the cerebrum of rats exposed to global cerebral ischemia and ischemia/ reperfusion. Eur Rev Med Pharmacol Sci. 2014;18:2387-92.

Copyright (c) 2019 The Author(s); Published by Nickan Research Institute. This is an open-access article distributed under the terms of the Creative Commons Attribution License (http://creativecommons.org/licenses/by/4.0), which permits unrestricted use, distribution, and reproduction in any medium, provided the original work is properly cited. 